\title{
Design of Fuzzy Damping Controller of UPFC through Genetic Algorithm
}

\author{
T. K. Mok* \\ Yixin Ni (Senior Member, IEEE) \\ Department of Electrical and Electronic Engineering \\ The University of Hong Kong, \\ Hong Kong \\ *E-mail: tkmok@eee.hku.hk
}

Felix F. Wu (Fellow, IEEE)

\begin{abstract}
The addition of a PSS-like supplementary controller to the UPFC main control can provide effective damping to the low frequency oscillation on the heavily loaded tie lines of interconnected power system. However, the conventional controller designed based on a linearized model cannot provide satisfactory performance over a wide range of operation point and under large disturbances. In this paper, the design of the Fuzzy Damping Controller (FDC) for UPFC as a substitute of the conventional supplementary controller is presented. Scaling factors are inserted to the membership functions of both inputs and output for easy design of fuzzifier and defuzzifier. Genetic Algorithm (GA) is applied to optimize the performance of the FDC through fine-tuning of the scaling factors. A 4-machine interconnected power system is used as the test system with a UPFC installed in one of the tie lines. Comparisons between the performances of both the fuzzy and the conventional supplementary controllers are studied. Computer test results show that FDC is very effective in damping the oscillation and in the meantime has a better robustness as compared with its conventional counterpart.
\end{abstract}

Keywords: Power system stability and control, FACTS, UPFC, fuzzy logic control, genetic algorithms

\section{INTRODUCTION}

In recent years, the progress in high power semiconductor device has stimulated the development of a new application in power system known as the flexible ac transmission system (FACTS). Application of FACTS to power system can enhance the controllability and flexibility of the whole system via the application made up of compact power electronic components $[1,2]$. The unified power flow controller (UPFC) is the most versatile and powerful device among the FACTS-device family. It can operate as a shunt and/or series compensator, a power flow controller, a voltage regulator or a phase shifter depending on its main control strategy. In this way, simultaneously control on bus voltage and transmission line power flow can be realized. The development of the first industrial UPFC is implemented under joint sponsorship of the EPRI, the AEP and the Westinghouse Electric Corporation of USA [3].
A UPFC power frequency model is suggested in [4], which provides an interface of the UPFC to the quasi-steady-state model of the transmission network for analyzing the effects of UPFC on large-scale power system stability. A versatile FACTS-device-ac-network interface is derived in [5] to participate the UPFC model into the conventional transient stability analysis program with good convergence and accuracy in time simulation. Investigation on the UPFC main control effects shows that the UPFC can improve the system transient stability and enhance the system transfer limit noticeably. The application of the UPFC to the modern power system can therefore lead to more flexible, secure and economic operation.

The UPFC can also increase the damping of the system through its supplementary control [4]. As the modern power systems are interconnected, usually low frequency oscillation will occur on heavily loaded tied line after a disturbance. Sometimes, the power system stabilizer (PSS) on a specific generator cannot provide effective damping for this kind of oscillation. In [4], it is shown that the addition of a PSS-liked supplementary controller (SC) can provide an effective solution to the problem. However, the conventional controller designed based on a linearized system model around a certain operation point cannot provide satisfactory performance over a wide range of operation conditions and under large disturbances.

In this paper, we attempt to design a fuzzy logic based controller to replace the conventional one proposed in [4]. Fuzzy logic control has a number of distinguished advantages over the conventional one [6]. It is not so sensitive to the variation of system structure, parameters and operation points and can be easily implemented in a largescale nonlinear system. Most importantly, human expert knowledge can be incorporated to the controller design easily. Many applications of the fuzzy logic theory have been seen in power engineering field [7].

Many of the fuzzy controllers rely on manual tuning of the parameters to obtained desired performance for a specific system. However, this may be very time consuming and reach a local-optimum. In this paper, we try to optimize the parameters of the fuzzy controller by using genetic algorithm (GA). GA is a very powerful optimization tool, which can provide global optimal solutions to any type of optimization problem. 
In section II, the power frequency model of the UPFC, the conventional main and supplementary control and the UPFC interface to ac network is presented. The structure and the operation principle of the fuzzy controller and the formulation for its parameter optimization by GA are covered in III. Computer tests on a multi-machine interconnected power system are conducted with the results shown in section IV. Conclusions are drawn in section V.

\section{UPFC MATH MODEL AND ITS CONVENTIONAL CONTROL}

\section{A. UPFC Power Frequency Model}

The power frequency model for the UPFC suggested in [4] is used in the paper and it is outlined as follow. Fig. 1 shows a schematic diagram for UPFC, where $n_{1}, X_{t 1}$ and $n_{2}, X_{t 2}$ are the voltage ratio and the reactance of the shunt and series transformers respectively. All the variables used in the UPFC model are denoted in Fig. 1 with bold fonts representing phasors. The ac system uses per unit system with its variables calculated based on the system-side $S_{B}$ and $V_{B}$, while the de variables are expressed in SI units.

The UPFC dc link capacitor dynamics can be expressed as follows with harmonics and UPFC losses neglected:

$$
C V_{d} \frac{d V_{d}}{d t}=\left(P_{1}-P_{2}\right) S_{B}
$$

where

$$
\begin{aligned}
& P_{1}=\operatorname{Re}\left(\dot{V}_{1} \dot{I}_{1}^{*}\right)=\operatorname{Re}\left(\dot{V}_{1}\left(\frac{n_{1} \dot{V}_{S}-\dot{V}_{1}}{j X_{t 1}}\right)^{*}\right) \\
& P_{2}=\operatorname{Re}\left(\dot{V}_{p q} \dot{I}_{L}\right)=\operatorname{Re}\left(\dot{V}_{p q}\left(\frac{\dot{V}_{S}+\dot{V}_{p q}-\dot{V}_{R}}{j X_{12}}\right)^{*}\right)
\end{aligned}
$$

PWM control technique is applied to both of the voltage source converters so that the relations between the inverter dc- and ac-side voltages is:

$$
\left\{\begin{array}{l}
V_{1}=m_{1} V_{d} / V_{B} \\
V_{p q}=m_{2} V_{d} /\left(V_{B} n_{2}\right)
\end{array}\right.
$$

where coefficients $m_{1}$ and $m_{2}$ represent the PWM control effects [11] in order to maintain desired inverter ac-side voltage $V_{1}$ and $V_{2}$ respectively.

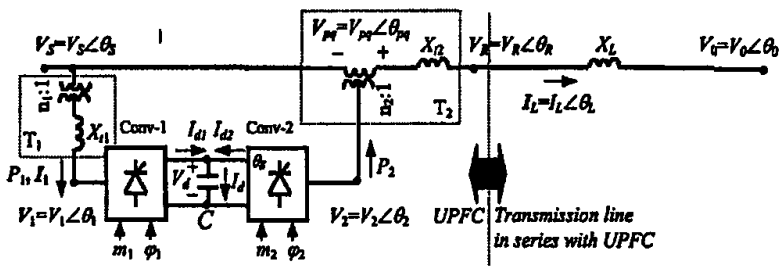

Fig. 1 Transmission line with UPFC installed
The phase angles of $V_{1}$ and $V_{2}$ can be controlled through firing angles $\varphi_{1}$ and $\varphi_{2}$ of the converters, i.e.

$$
\left\{\begin{array}{l}
\theta_{1}=\theta_{S}-\varphi_{1} \\
\theta_{p q}=\theta_{S}-\varphi_{2}
\end{array}\right.
$$

The preferred $m_{1}, m_{2}, \varphi_{1}$ and $\varphi_{2}$ are outputs from the UPFC main control which are assumed to be realized without time deliyy. Equations (1) to (4) constitute the UPFC power frequency model for power system stability study.

\section{B. UPFC Conventional Main and Supplementary control}

The UPFC shunt and series element conventional main control and supplementary control diagrams are shown in Fig. 2-4 respectively.

The shunt element control is the constant dc link capacitor voltage control realized by controlling the firing angle $\varphi_{1}$ of inverter 1 (Fig. 2(a)); and the constant ac bus voltage control achieved by controlling $m_{1}$ of the PWM controller of inverter 1 (Fig. 2(b)). Although a simple transfer function is used for the main control, there is no difficulty to include more complicated transfer functions. As for the series element, the inserting voltage $\dot{V}_{p q}$ in Fig. 1 can be

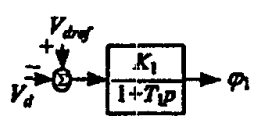

(a)

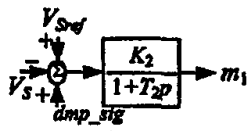

(b)
Fig. 2 UPFC shunt element main control diagram

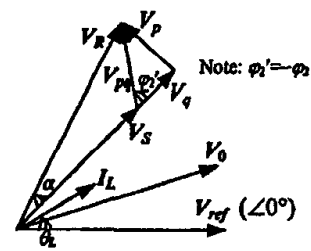

(a)

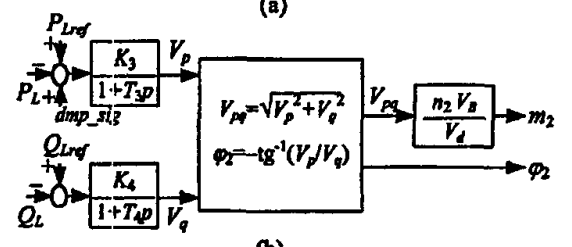

(b)

Fig. 3 UPFC series element main control and the phasor diagram

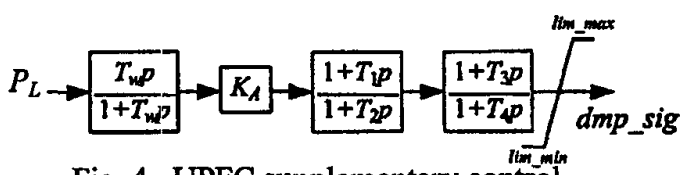

Fig. 4 UPFC supplementary control 
decomposed to $V_{p}$ and $V_{q}$. The former is perpendicular to $\dot{V}_{S}$, and the latter is in phase with $\dot{V}_{S}$ (see Fig. 3(a)).

Obviously, these two components have strong impacts on active and reactive power flow respectively. Based on this decomposition, Fig. 3(b) shows the constant active and reactive power flow control for the series element main control.

In order to improve system damping, a PSS-like supplementary control [12] (see Fig. 4) is added with its output $d m p$ sig used to modulate the terminal bus voltage (Fig. 2(b)) or the tie line real power (Fig. 3(b)) to provide damping effects. In this paper the bus voltage modulation approach is used.

\section{Mathematical model for other elements and the interface of the UPFC to ac network}

The subtransient model is used for the generators with the third-order excitation control [12]. The mechanical power of each generator is approximately constant. Loads are expressed as constant impedance and the ac network is linear. The sequential solution method, which has been successfully used in ac/dc power system load flow and transient stability analysis for ac-dc interface, is extended for UPFC interface to ac network. The detail can be found in [5].

\section{FUZZY DAMPING CONTROLLER (FDC)}

In this section, a fuzzy supplementary controller is designed for UPFC to damp power oscillation. Fig. 5 shows the block diagram of the proposed Fuzzy Damping Controller (FDC). It is indeed a fuzzy PI controller. The FDC input signal is same as its conventional counterpart; i.e., the tie line real power $P_{L}$. The FDC output is still the damping signal dmp_sig, which will be sent to the main controller for damping power oscillation.

\section{A. Structure of the designed FDC}

The two gains (or scaling factors) $K_{1}$ and $K_{2}$ are inserted to make $x_{1}^{\prime}=K_{1} x_{1}$ and $x_{2}^{\prime}=K_{2} x_{2}$ usually at a proper range of [$1,1]$ for easy design of the fuzzifier. The gain $K_{3}$ at the output path plays a similar role for easy design of the defuzzifier. $K_{1}, K_{2}$ and $K_{3}$ should be optimised in order to get satisfied FDC performance.

The differentiable Gaussian membership function is used to fuzzifying the two input signals. The triangular function is used to defuzzify the output fuzzy sets for its easy calculation. Seven fuzzy sets are defined for each of the input and output signals. They are NB, NM, NS, ZR, PS, PM and PB (see Fig. 6(a) and (b)) which stand for negative big, negative medium, negative small, zero, positive small, positive medium and positive big respectively. In the implementation, $\mathrm{PB}$ and $\mathrm{NB}$ are extended to reach \pm 2 respectively so as to fit the input signals of excessive range. The fuzzy rule base is formed based on experiences. Table 1 shows the fuzzy rules which take the form:

\section{IF $K_{I} x_{1}$ is $A_{i}$ and $K_{2} x_{2}$ is $B_{i}$ THEN output $y / K_{3}$ is $C_{l}$}

where $A_{i}$ and $B_{i}$ are the input fuzzy sets with Gaussian membership functions, while $C_{i}$ is the output fuzzy set with triangular membership function.

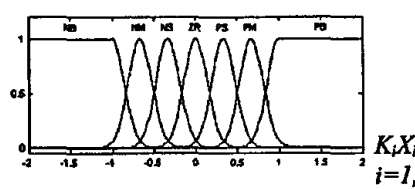

(a)

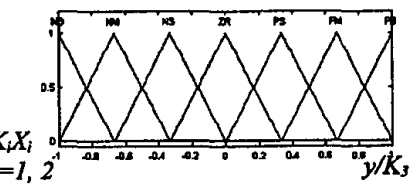

(b)
Fig. 6 Membership Functions for (a) input and (b) output signals

In the FDC, singleton fuzzifier, centre average defuzzifier, and product inference engine are used for fuzzifying, defuzzifying and inference process respectively

In order to obtain the optimized FDC performance the FDC coefficients should be well designed. It has been found that the gain $K_{i}$ has more significant impacts on FDC performance than other parameters. Therefore how to tune $K_{i}$ becomes the key in FDC design. Genetic algorithm (GA)

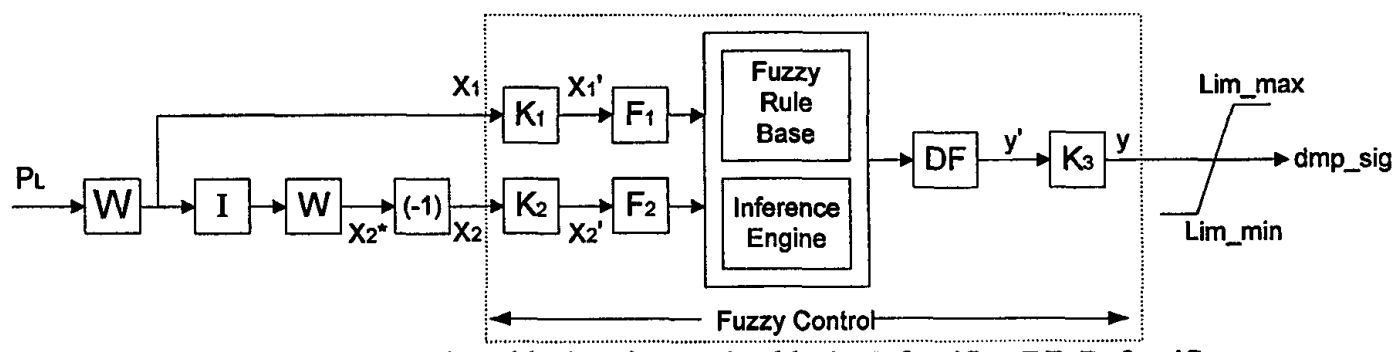

W: washout block, I: integration block, F: fuzzifier, DF: Defuzzifier

Fig. 5 Fuzzy damping controller for the UPFC 
is used in FDC parameter optimization as describe below.

Table 1 Rule-base table

\begin{tabular}{|c|c|c|c|c|c|c|c|}
\hline $\begin{array}{c}K_{1} x_{1} \\
K_{2} x_{2}\end{array}$ & NB & NM & NS & ZR & PS & PM & PB \\
\hline NB & PB & PB & PB & PM & PM & PS & ZR \\
\hline NM & PB & PB & PM & PM & PS & ZR & NS \\
\hline NS & PB & PM & PM & PS & ZR & NS & NM \\
\hline ZR & PM & PM & PS & ZR & NS & NM & NM \\
\hline PS & PM & PS & ZR & NS & NM & NM & NB \\
\hline PM & PS & ZR & NS & NM & NM & NB & NB \\
\hline PB & ZR & NS & NM & NM & NB & NB & NB \\
\hline
\end{tabular}

\section{B. Genetic Algorithm for FDC Coefficient Optimization}

Genetic algorithm (GA) is a promising approach for the optimization of $K_{i}$. It performs multi-directional search of the solution space by reproduction while keeping the population size constant for all generations.

The optimization problem considered here is to search the optimal set of the controller scaling factors $K_{1}$ which can provide the best damping to the system. The optimization of the FDC by GA begins with a random set of initial population. Floating point number is used to represent the $K_{i}$ for faster convergence and better precision. An individual solution, denoted as a chromosome, in a population is composed of the three scaling factor $K_{1}, K_{2}$ and $K_{3}$. Initial simulations are conducted to select the feasible solution space of the problem so as to increase the convergent rate.

For each of the individual, time simulation is conducted to obtain the transient response of the system to a certain disturbance. The fitness of the individual is then evaluated based on the equation:

$$
J=\sum_{j=1}^{M} \sum_{k=1}^{N}\left|\Delta \delta_{j}(k)\right|
$$

where $M$ is the number of key machines which are strongly participating to the power oscillation to be damped by the FDC and $N$ is the number of simulation time steps in a specific time interval. Equation (6) measures the rotor swing of the key machines within the dominant oscillation time after a large disturbance corresponding to a certain set of $\left\{K_{i}\right\}$.

Genetic operations, crossover and mutation take place to reproduce the offspring from the parents $[8,9]$. Crossover is the main operator of GA. It allows the mates exchange information with each other by combining two individuals' features to form a new individual. Arithmetic crossover is used in this paper, which is defined as follows:
If $v_{1}$ and $v_{2}$ ari chosen to perform crossover, the resulting offspring are:

$v_{1}^{\prime}=r \cdot v_{1}+(1-r) \cdot v_{2}$

$v_{2}^{\prime}=(1-r) \cdot v_{1}+r \cdot v_{2}$

where $r$ is a random number between 0 and 1 .

On the other hand, mutation introduces new solution to the population for trail by producing spontaneous random change in various individuals. Non-uniform mutation are used in this work and is defined as follows:

If an element $v_{k}$ of a parent $v$ is selected for mutation, the result would be:

$v_{k}^{\prime}= \begin{cases}v_{k}+\left(U B-v_{k}\right) f(t) & \text { If a random digit is 0 } \\ v_{k}-\left(v_{k}+L B\right) f(t) & \text { If a random digit is } 1\end{cases}$

where UB and LB are the upper and lower bounds of the variable $v_{k}$ respectively, $t$ is the current generation. The function $f(t)$ should return a value in the range $[0,1]$ such that the probability of $f(t)$ being close to 0 increases as $t$ increases. This: ensures the operator to search the space uniformly initially and very locally at later generations. The following function is used:

$$
f(t)=\left(r \cdot\left(1-\frac{t}{T}\right)\right)^{b}
$$

where $r$ is a random number in the range $[0,1], T$ is the maximum nurnber of generation, and $b$ is a system parameter determining the degree of non-uniformity, which is taken to be 3 here.

Successive generations are produced by selecting fitter chromosomes from the preceding generation. Fitter chromosomes, i.e. those with smaller index $J$, have a higer chance to be selected. Roulette wheel selection is used to perform the evolution operation. The process repeats until a specific maximum number of generation is reached. The chromosome with the smallest value of $J$ is taken as the final solution.

\section{COIMPUTER SIMULATION RESULTS}

The two-area 4-machine interconnected power system shown in Fig. 7 is used as the test system with a UPFC installed in one of the tie line 101-13. At the steady state, about $700 \mathrm{MW}$ power is generated from each of the generators. The loads on buses 3 and 13 are $967 \mathrm{MW}$ and $1767 \mathrm{MW}$ respectively. About $400 \mathrm{MW}$ power is transferred from area 1 to area 2 through the parallel tie lines. The 
parameters of the UPFC system and its main control can be found in appendix.

\section{A. Optimization result from $G A$}

A three-phase to earth fault at line 3-101 close to terminal 3 with duration of $0.1 \mathrm{~s}$ is applied to the systems at full load as the disturbance to the system. The performance index $J$ of each chromosome is evaluated based on the transient response of the system. As the machine swing in the first $3 \mathrm{~s}$ after the disturbance would contribute a large portion to the index, the response in the first $3 \mathrm{~s}$ is discarded in the evaluation of $J$ to avoid immature convergence. A time step of $0.005 \mathrm{~s}$ is used and the system response up to $7 \mathrm{~s}$ is considered. Accordingly, (6) becomes:

$$
J=\sum_{j=1}^{M} \sum_{k=600}^{1400}\left|\Delta \delta_{j}(k)\right|
$$

The population size and number of generations for the GA are chosen as 30 and 100 respectively. The optimized $K$ is:

$$
K_{G A}=(0.56,1.73,0.68)
$$

The convergence rate of the performance index throughout the 100 generations is shown in Fig. 8.

\section{B. Comparison of the dynamic performances of the designed FDC and CSC}

The performance of the designed FDC is evaluated through time simulation, with the response of the CSC given for comparison purpose. A three-phase fault on tie line 3-101 close to the terminal bus 3 is considered. The fault occurs at $t=0.5 \mathrm{~s}$ and it is cleared in $0.1 \mathrm{~s}$. Fig. 9 shows the rotor angle oscillation of the machine 1 after the disturbance for various cases. It can be observed that when there is no supplementary controller installed to the UPFC, the damping of the system is very poor. The addition of either the CSC or the FDC can increase the system damping significantly and reduce the first swing peak angle slightly. The result also shows that the performance of FDC is much better than the CSC.

The loads on buses 3 and 13 are changed gradually to test the performance of the controllers on the different operating points. The parameters of all controllers are remained unchanged. Using the same disturbance as before, the performance indexes calculated based on (10) are listed in Table 2. While the operation point of the system is changing, the FDC still performs very well as compared with the CSC. For each of the cases, in term of the performance index $J$, the performance of FDC is at least $30 \%$ better than that of the CSC.
Table 2 - Performance Index $J$

\begin{tabular}{c|ccc}
\hline Load & No SC & CSC & $\begin{array}{c}\text { FDC } \\
\text { (GA) }\end{array}$ \\
\hline $110 \%$ & 1734.4 & 750.8 & 424.8 \\
$100 \%$ & 1144.5 & 461.1 & 303.4 \\
$90 \%$ & 901.5 & 371.8 & 248.0 \\
$80 \%$ & 675.1 & 314.6 & 217.0 \\
\hline \multicolumn{3}{c}{ V CONCLUSION }
\end{tabular}

An advanced Fuzzy Damping Controller (FDC) is designed for UPFC to damp the low frequency oscillation in the tie line of the interconnected systems. Genetic Algorithm (GA) is used to optimize the parameters of the FDC. The computer test results show that the FDC is more effective in damping the oscillation and possesses better robustness as compared with its conventional counterpart.

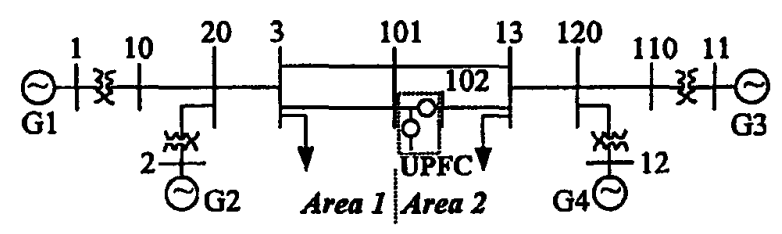

Fig. 7 Two-area 4-machine test system

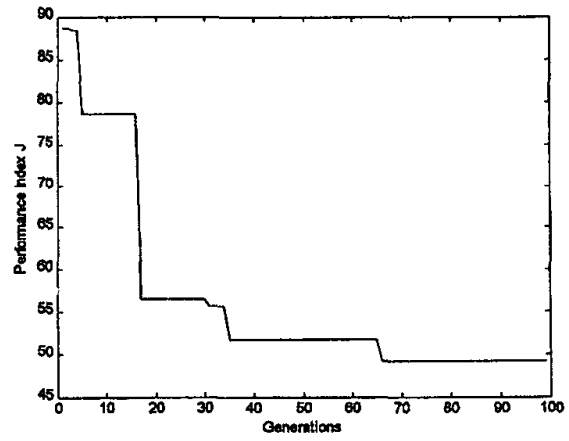

Fig. 8 Evolution of Performance Index $J$ machine 1 rotor angle variation

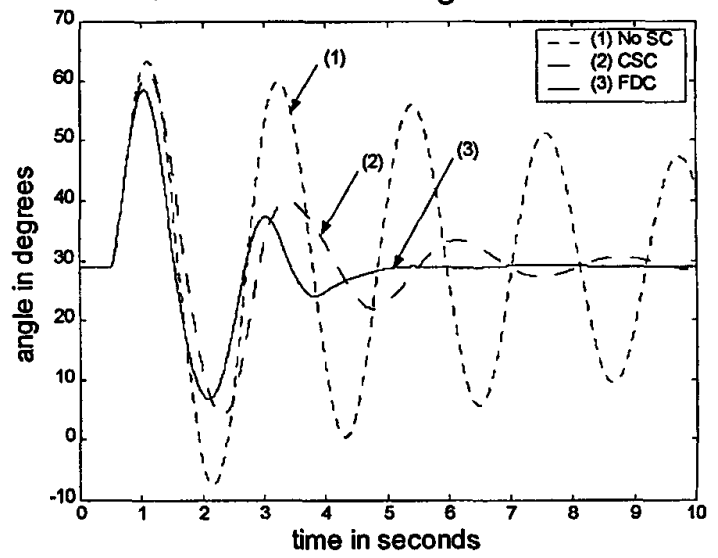

Fig. 9 System responses at full load 


\section{ACKNOWLEDGEMENT}

This project is jointly sponsored by the National Key Basic Research Special Fund (No. G1998020305), RGC (Hong Kong) and EPRI (USA), to whom the authors would like to express their sincere acknowledgement.

\section{REFERENCE}

[1] IEEE FACTS Application Task Force, "FACTS Applications", Publication of IEEE PES Summer Meeting, 1996.

[2] L. Gyugyi, "Unified Power-Flow Control Concept for Flexible AC Transmission Systems", IEE Proceedings-C, Vol. 139, No.4, July 1992. pp: 323-331

[3] A.S. Mehraban, A.J.F. Keri, et al., "Application of the World's First UPFC on the AEP System", EPRI-the Future of Power delivery Conference, Washington DC, 1996.

[4] Zhenyu Huang, Yixin Ni, et al. "Application of Unified Power Flow Controller in Interconnected Power Systems -Modeling, Interface, Control Strategy and Case Study", Accepted for Publishing on IEEE Transaction on Power Systems

[5] Lai On Mak, Zhenyu Huang, Yixin Ni, et al, " A versatile interface between FACTS devices and ac power systems", Proceedings of 13th Power System Computation Conference, Norway, 1999. pp: 533-538

[6] Li-Xin Wang, "A Course in Fuzzy Systems and Control", Prentice Hall, 1997.

[7] J. A. Momoh, and X. W. Ma, et al. "Overview and Literature Survey of Fuzzy Set Theory in Power Systems", IEEE Transaction on Power Systems, Vol. 10, No. 3, Aug. 1995. pp: $1676-1690$

[8] Z. Michalewicz, "Genetic Algorithms + Data Structures = Evolution Programs", Springer, 1996

[9] M. Gen and R. Cheng, "Genetic Algorithms and Engineering Design", Wiley, 1997

[10] T.K. Mok, Zhenyu Huang, Yixin Ni, et al, " Design of a Fuzzy Damping Controller for UPFC through Gradient Descent Training", paper submitted to International Journal of EPSR.

[11] N. Mohan, T. M. Undeland and W. P. Robbins, Power Electronics: Converters, Applications and Design, Second Edition, John Wiley \& Sons, 1995.

[12] P. Kundur, "Power System Stability and Control", McGraw Hill, 1993.

\section{APPENDIX}

Parameters of the UPFC and its control system UPFC parameters:

$V_{B}=220 \mathrm{kV}, S_{B}=100 \mathrm{MVA}, n_{1}=0.05, n_{2}=0.25, X_{t 1}=0.00025$, $X_{R}=0.05$ (all in p. u.)

The parameters for UPFC main control:

$K_{1}=0.05, T_{1}=0.01, K_{2}=1.0, T_{2}=0.05, K_{3}=5.0, T_{3}=0.1, K_{4}=5.0$,

$T_{4}=0.1, V_{\text {d,ref }} \approx 44 \mathrm{kV}$

The parameters of the CSC:

$K_{A}=0.83, T_{w}=3.0 \mathrm{~s}, T_{1}=T_{3}=0.02 \mathrm{~s}, T_{2}=T_{4}=0.33 \mathrm{~s}$, lim_max $=0.2$,

lim_min $=-0.2$
Membership functions for FDC

Gaussian membership function:

$\mu_{G}(x)=\exp \left(-\left(\frac{x-\bar{x}}{\sigma}\right)^{2}\right)$

where the parameters $[\sigma, \bar{x}]$ for the different fuzzy sets are:

'NB': [0.1415-1], 'NM': [0.1415 -0.6667],

'NS': [0.1415 -0.3333], 'ZR': [0.1415 0],

'PS': [0.1415 0.3333], 'PM': [0.1415 0.6667]

'PB': [0.1415 1].

Triangular membership function:

$\mu_{T}(x)= \begin{cases}0, & x \leq a \\ \frac{x-a}{b-a}, & a \leq x \leq b \\ \frac{c-x}{c-b}, & b \leq x \leq c \\ 0, & x \geq c\end{cases}$

where the parameters $[a, b, c]$ for the different fuzzy sets are:

'NB': [-1.3333 -1 -0.6667], 'NM': [-1 -0.6667 -0.3333],

'NS': [ $\left.\begin{array}{lllll}-0.6667 & -0.3333 & 0\end{array}\right], \quad$ 'ZR': $\left[\begin{array}{llll}-0.3333 & 0 & 0.3333\end{array}\right]$,

'PS': [ $\left.\begin{array}{lll}0 & 0.3333 & 0.6667\end{array}\right], \quad$ 'PM': [0.3333 0.6667 1],

'PB': [0.6667 1 1.3333].

\section{BIOGRAPHIES}

T.K. Mok received his B.Eng. degree in Electrical Energy Systems Engineering from. The University of Hong Kong in 1998 with a first class honor. He is now a M. Phil. candidate in HKU.

Yixin $\mathrm{Ni}$ received her B.Eng., M. Eng., and Dr. Eng. from Electrical Engineering Department, Tsinghua University, P.R. China in 1968, 1981 and 1983 respectively. Her research interests are in power system modeling, simulation, stability and control and power electronicis applications in power systems. She was a professor of Tsinghua University and is now with The University of Hong Kong.

Felix F. Wu joined The University of Hong Kong as Chair professor of Electrical Engineering in September 1995 and is now Pro-vice-chancellor of HKU. Prior to that, he was Professor and Vice Chairman, Department of Electrical Engineering and Computer Sciences, University of California, Berkeley, the same institute where he received his $\mathrm{Ph} . \mathrm{D}$. degree. His research interests are in power system planning and operation, including economics and reliability in system planning, real-time security assessment, and design of energy management systems and distribution automation. Recently he has been involved in the design of industry restructuring and electricity pricing. 